\title{
Fatal Serratia marcescens meningitis and myocarditis in a patient with an indwelling urinary catheter
}

\author{
J S Johnson, J Croall, J S F Power, G R Armstrong
}

\begin{abstract}
Serratia marcescens is commonly isolated from the urine of patients with an indwelling urinary catheter and in the absence of symptoms is often regarded as a contaminant. A case of fatal Serratia marcescens septicaemia with meningitis, brain abscesses, and myocarditis discovered at necropsy is described. The patient was an 83 year old man with an indwelling urinary catheter who suffered from several chronic medical conditions and from whose urine Serratia marcescens was isolated at the time of catheterisation. Serratia marcescens can be a virulent pathogen in particular groups of patients and when assessing its significance in catheter urine specimens, consideration should be given to recognised risk factors such as old age, previous antibiotic treatment, and underlying chronic or debilitating disease, even in the absence of clinical symptoms. (f Clin Pathol 1998;51:789-790)
\end{abstract}

Keywords: Serratia marcescens; meningitis; myocarditis; catheter urine

Serratia marcescens is a Gram negative bacillus, a member of the Enterobacteriaceae, which is distributed widely in the environment. Initially considered as a non-pathogenic saprophyte, it is now well known as a cause of urinary tract infections, wound infections, meningitis, pneumonia, septicaemia, and endocarditis. ${ }^{1}$ It is commonly isolated from the urine of patients with indwelling urinary catheters and is widely considered to be only of significance in patients with serious underlying disease, particularly neonates, patients requiring treatment on intensive care units, neutropenic patients, or those with disseminated malignancy. We report a case of fatal bacterial meningitis, cerebral abscesses, and myocarditis caused by $S$ marcescens in an elderly man with an indwelling urinary catheter to highlight the risk factors for virulence in this clinical situation.

\section{Case report}

An 83 year old man was admitted with a two day history of dyspnoea and orthopnoea. He had a past medical history of ischaemic heart disease with episodes of left ventricular failure, chronic obstructive airways disease, and chronic renal impairment owing to obstructive nephropathy secondary to benign prostatic hyperplasia. He had undergone two transurethral resections of the prostate gland six and seven years previously and did not require a urinary catheter. On examination he was apyrexial, tachypnoeic, and hypertensive with a blood pressure of $170 / 110 \mathrm{~mm} \mathrm{Hg}$. He had bilateral crepitations and a chest $x$ ray suggested pulmonary oedema and consolidation. Culture of sputum taken on admission was negative. He was treated with intravenous diuretics and intravenous cefuroxime $750 \mathrm{mg}$ three times daily. His breathlessness improved on this regimen but his renal function deteriorated and he became dehydrated, requiring intravenous fluid replacement. His renal function continued to deteriorate and he developed peripheral oedema which involved the penis to the extent that he required urinary catheterisation to prevent occlusion of the external urinary meatus.

At the time of catheterisation, 18 days after admission, a specimen of urine was taken which contained more than $100 \times 10^{6} /$ litre white blood cells, no red cells, and had a total bacterial count of more than $100 \times 10^{6} /$ litre. Serratia marcescens was identified by means of API 20e (bioMerieux) which was sensitive to trimethoprim, gentamicin, nalidixic acid, and ciprofloxacin and resistant to ampicillin, nitrofurantoin, and cephalexin.

The patient was clinically asymptomatic and the bacteriuria was not thought significant by the laboratory since it was catheter associated, so antibiotic treatment was not begun. One week after admission his white cell count was $8.3 \times 10^{9} /$ litre and four days before discharge it was $11.5 \times 10^{9} /$ litre. For the duration of his admission he remained apyrexial. The patient's congestive heart failure improved and his renal function returned to its original level. During the last 10 days in hospital he had episodes of diarrhoea and was noticed to be lethargic with intermittent confusion and restlessness. Five weeks after admission he was discharged to a nursing home with a long term urinary catheter in place. He died the day after discharge and a necropsy was performed.

Pathological examination

At necropsy there was a purulent meningitis, particularly over the posterior cerebellum. There were multiple abscesses scattered throughout the brain, especially in the grey matter of the occipital lobes. The heart was of normal weight and the cardiac valves were normal but the endocardium was studded with multiple pustules measuring up to $3 \mathrm{~mm}$, and the interventricular septal myocardium contained several pale pink abscesses measuring
Accepted for publication 28 May 1998 


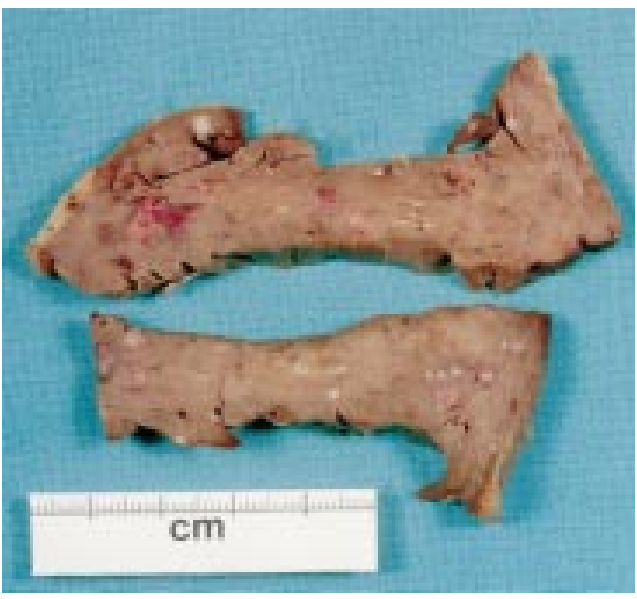

Figure 1 Sections of formalin fixed myocardium containing multiple abscesses with pink coloration typical of Serratia marcescens infection.

up to $4 \mathrm{~mm}$ (fig 1 ). Both lungs were congested but showed no evidence of consolidation or abscess formation. The kidneys were shrunken and showed severe cortical atrophy and calyceal blunting. The bladder showed focal mucosal congestion. There was a large infrarenal abdominal aortic aneurysm and severe complicated atherosclerosis of the remainder of the aorta. No evidence of malignancy or lymphoproliferative disease was present at necropsy.

Postmortem culture of the meningeal pus produced a pure isolate of Serratia marcescens. Histology confirmed the presence of a purulent meningitis and multiple brain abscesses, predominantly disposed around blood vessels consistent with haematogenous spread (fig 2). The cerebral and myocardial abscesses contained colonies of Gram negative organisms. There was evidence of chronic myocardial ischaemia in the form of interstitial fibrosis, and the kidneys showed chronic ischaemic damage, hypertensive vessel changes, and occlusion of several vessels by cholesterol emboli but no evidence of an acute pyelonephritis.

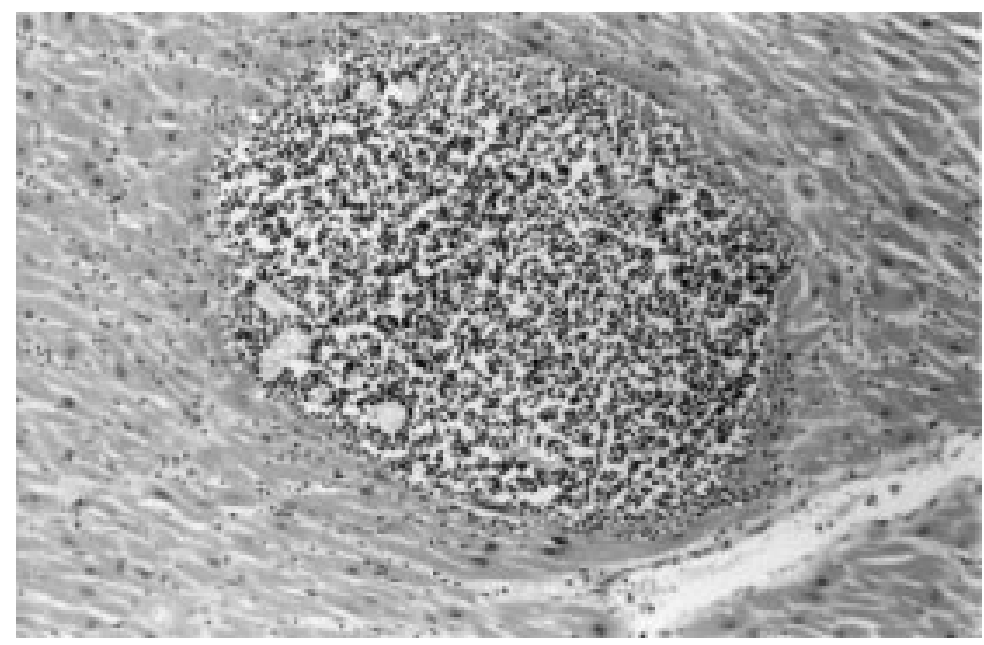

Figure 2 Myocardium showing abscess formation with peripheral myocyte necrosis. (Haematoxylin and eosin stain; objective $\times 40$.)

\section{Discussion}

Serratia marcescens is commonly isolated from the urine of hospital inpatients, particularly those with a history of an indwelling urinary catheter, malignancy, recent surgery, or antibiotic treatment, and has on occasion been responsible for nosocomial outbreaks. ${ }^{2}$ Most cases of clinically significant $S$ marcescens bacteraemia occur in surgical patients or in those on the intensive care unit. Haddy et al examined the underlying diseases present in patients with $S$ marcescens bacteraemia, in whom the mortality was $25 \%$, and found that $20 \%$ had advanced malignancy, $13 \%$ acute or chronic renal failure, $10 \%$ diabetes mellitus, $7 \%$ chronic obstructive pulmonary disease, and $7 \%$ were postoperative cases. ${ }^{3}$ Interestingly, no pyogenic metastatic foci were noted in the fatal cases in this study. Another study ${ }^{4}$ looked at 48 patients with $S$ marcescens infections and found the majority were elderly males with chronic debilitating diseases who had received antibiotics before the isolation of serratia and who had indwelling catheters inserted during their hospital admission. Eleven of these patients died and in six of these, including three cases of fatal septicaemia, the organism appeared to play a role in the outcome. These predisposing factors are similar to those defined in a study of 23 cases of Serratia marcescens bacteraemia in which all six fatal cases were medical patients. ${ }^{5}$ Patients bacteraemic with $S$ marcescens commonly do not have either a leucocytosis or a pyrexia. ${ }^{35}$ $S$ marcescens has been described as causing endocarditis with contiguous myocarditis in patients with valvar heart disease undergoing urological procedures ${ }^{6}$ and in intravenous drug addicts. ${ }^{1}$ To our knowledge it has not been reported as causing a multifocal septic myocarditis without valvar vegetations as was present in our case. The striking pink coloration of the abscesses noted at necropsy is characteristic of $S$ marcescens infection, and is caused by prodigiosin, a biosynthesised pigment unique to this organism and used as a biological marker in the past. ${ }^{7}$

Our case emphasises that the threshold for assigning significance to $S$ marcescens isolates from catheter specimens of urine should be lowered, even in the absence of clinical symptoms, if the patient displays certain risk factors such as old age, underlying chronic or debilitating disease, or previous antibiotic treatment.

1 Hejazi A, Falkiner FR. Serratia marcescens. $\mathcal{f}$ Med Microbiol 1997;46:903-12.

2 Madduri SD, Mauriello DA, Smith LG, et al. Serratia marcescens and the urologist. F Urol 1976;116:613-15.

3 Haddy RI, Mann BL, Nadkarni DD, et al. Nosocomial infection in the community hospital: severe infection due to serratia species. F Fam Prac 1996;42:273-7.

4 MacArthur BS, Ackerman NB. The significance of Serratia as an infectious organism. Surg Gynaecol Obstet 1978;146: 49-53.

5 Wong WW, Wang LS, Cheng DL, et al. Serratia marcescens bacteraemia. F Formosan Med Assoc 1991;90:88-93.

6 Marier R, Valenti AJ, Madri JA. Gram-negative endocarditis following cystoscopy. F Urol 1978;119:134-7.

7 Yu VL. Serratia marcescens: historical perspective and clinical review. N Engl f Med 1979;300:887-93. 